\title{
La pratique de l'intervention d'ergonomes consultants : une approche réflexive orientée par les outils
}

The practice of ergonomist consultant: a reflexive tools-based approach

La práctica de la intervención de los ergónomos consultores: une enfoque

reflexivo orientado por las herramientas

Léonard Querelle et Jean-François Thibault

\section{OpenEdition}

\section{Journals}

Édition électronique

URL : http://journals.openedition.org/activites/1458

DOI : 10.4000/activites. 1458

ISSN : $1765-2723$

Éditeur

ARPACT - Association Recherches et Pratiques sur les ACTivités

Référence électronique

Léonard Querelle et Jean-François Thibault, «La pratique de l'intervention d'ergonomes consultants une approche réflexive orientée par les outils », Activités [En ligne], 4-1 | avril 2007, mis en ligne le 15 avril 2007, consulté le 02 mai 2019. URL : http://journals.openedition.org/activites/1458; DOI : 10.4000/activites.1458

\section{(c) (i) (3) $\Theta$}

Activités est mis à disposition selon les termes de la licence Creative Commons Attribution - Pas d'Utilisation Commerciale - Pas de Modification 4.0 International. 


\title{
La pratique de l'intervention d'ergonomes consultants : une approche réflexive orientée par les outils
}

\author{
Léonard Querelle \& Jean-François Thibault \\ Laboratoire d'ergonomie des systèmes complexes (L.E.S.C.), Université Bordeaux II, France. \\ leonard.querelle@wanadoo.fr \\ Jean-Francois.Thibault@ergo.u-bordeaux2.fr
}

\begin{abstract}
The practice of ergonomist consultant: a reflexive tools-based approach

This article proposes to analyze the work of the ergonomist consultant in intervention on the basis of a service relationship model. The authors show that the activity of advising is based entirely on a service provision basis. From this point of view, it proves that the service relationship applied to the ergonomic intervention raises the issue of the objects used in the intervention. However, the theoretical outline of the service relationship must be adapted to study the ergonomic intervention. Thus we start from a reflexive point of view on the intervention to test the heuristic dimension of the model and to raise new issues.
\end{abstract}

KEYWORDS:

activity theory, ergonomist practice, service relationship, project control, ergonomic intervention

\section{Introduction}

$\mathrm{Au}$ quotidien, l'ergonome utilise des outils pour réaliser ses interventions. Si ces outils sont pour partie issus du patrimoine théorique et pratique de l'ergonomie, l'une des facettes du travail de l'ergonome est de les concevoir, de les adapter et de les optimiser de manière à gagner en performance.

Comme pour la production de biens, il nous semble important d'aborder la notion de performance d'une intervention ergonomique (Thibault, 2002) au travers des outils utilisés par l'ergonome consultant. Nous allons dans un premier temps aborder l'intervention ergonomique sous l'angle de la relation de service et montrer dans un deuxième temps en quoi les outils utilisés par l'ergonome consultant structurent son intervention.

\section{1.- Du conseil au modèle de la relation de service}

Gadrey (1994) évoque le conseil comme étant le plus extrême des services intangibles le considérant comme une activité relationnelle par excellence. Il le définit comme une activité qui consiste :

- à analyser un problème rencontré par une organisation ;

- à proposer des solutions ou recommandations fondées sur un diagnostic ;

- à participer à la mise en œuvre des solutions ;

De fait, le conseil relève du champ de la relation de service.

Si le fondateur de la relation de service semble être Goffman (1968), nous retenons cependant la définition, plus récente, proposée par Gadrey (cf. Figure 1). Il envisage la relation de service comme " une opération visant une transformation d'état d'une réalité $C$, possédée ou utilisée par un consommateur - client ou usager - B, réalisée par un prestataire $A$, à la demande de $B$ et souvent en relation 
avec lui, mais n'aboutissant pas à la production d'un bien susceptible de circuler économiquement, indépendamment du support $C »$ (Gadrey, 1992).

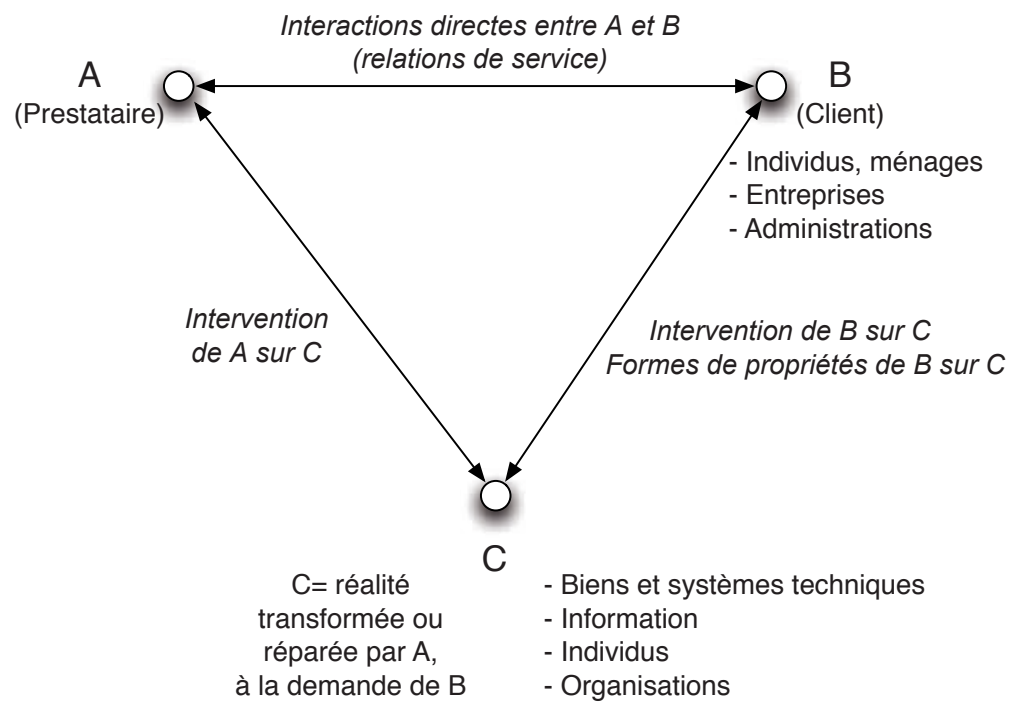

Figure 1 : Un modèle de la relation de service

De nouvelles perspectives s'ouvrent à partir des réflexions de Mayen (2005). En effet, le prestataire doit faire face à deux dimensions distinctes :

- l'objet sur lequel l'intervention doit porter ;

- la relation que l'usager entretient avec cet objet (souvent relation de dépendance).

De plus, l'engagement du prestataire implique une double activité au sein de situations de gestion (Jackson, 1998) :

- de son intervention sur l'objet mais aussi de l'intervention du client ;

- de la relation de service.

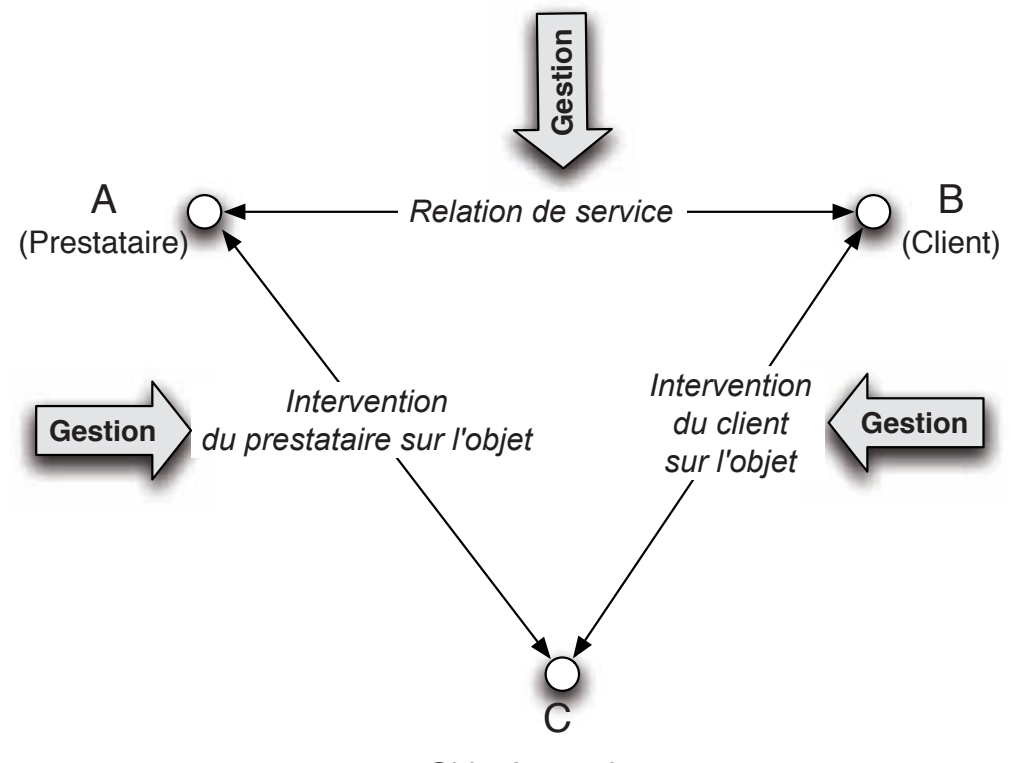

Objet à transformer

Figure 2: La gestion de relation de service

La représentation proposée, en Figure 2, est très proche du triangle de la consultation formalisée par 
Villette (2003, p. 51).

L'élément intéressant dans cette manière de décrire la relation de service repose sur le fait que l'un des objets du travail du prestataire puisse être la relation qu'entretient l'usager avec cet objet. L'hypothèse peut être alors formulée ainsi «non seulement les professionnels du service ont à construire un répertoire de connaissances portant sur l'objet technique du service, la relation entretenue par le client à l'objet et le cadre social et institutionnel dans lequel se déroule la transaction de service, mais aussi que ces trois domaines doivent être étroitement reliés en un système de représentation et d'action intégrateur $\gg$ (Mayen, 2005, p. 63).

Autrement dit, le client du service s'inscrit dans un contexte socioéconomique et institutionnel qui influence son action stratégique. D'une part, le prestataire doit donc comprendre l'ensemble des caractéristiques de cet environnement pour agir. D'autre part il dépend, lui-même, d'éléments extérieurs relatifs à son propre contexte socioéconomique et institutionnel. Ces contraintes imposées par les contextes du client et du prestataire participent à la construction d'un cadre au même titre qu'une prescription. Le consultant doit apporter la même vigilance à son intervention sur l'objet qu'à l'intervention du client sur ce même objet.

\section{2.- « Système de relations de services " : perspectives pour l'intervention ergonomique}

À partir des limites du modèle précité de la relation de service, nous allons envisager un modèle d'intervention ergonomique basé sur un système de relations de services. Bien évidemment, ce système évolue dans le temps comme les objets intermédiaires utilisés par l'ergonome consultant.

\section{1.-Les limites du modèle de la relation de service appliqué à l'intervention ergonomique}

Les invitations à regarder la pratique des ergonomes en France sous l'angle des relations de service se multiplient (Hubault, 2001 ; Cerf, \& Falzon, 2005 ; Petit, 2005). A partir de ces travaux et de notre pratique réflexive d'ergonome consultant, nous allons exposer les limites et proposer de nouvelles perspectives de modélisation ${ }^{1}$.

L'application du modèle précédemment présenté (cf. Figure 2) à l'intervention ergonomique conduit à une nouvelle relecture de ce modèle. Le prestataire devient l'ergonome consultant et l'objet à transformer devient un système sociotechnique ${ }^{2}$ quelle que soit la focale retenue (poste de travail, atelier, usine, ...). À partir de la notion d'usager du service (que nous préférons à client qui reste trop limité à un rapport économique), le débat s'ouvre laissant apparaitre plusieurs interrogations.

En effet, qui peut-être l'usager du service « ergonomique »? Dans le secteur industriel, est-ce le directeur de l'entreprise, le président-directeur général, le responsable de production, le contremaître, l'opérateur, voire le consommateur? La question se pose tout autant dans les institutions publiques comme les hôpitaux : est-ce le directeur régional, le directeur de l'hôpital, l'ingénieur, le chef de service, le médecin, le cadre supérieur, l'infirmière, l'aide-soignant ou le patient ? Déjà un certain nombre de précautions semblent nécessaires quant à l'identification d'un des trois pôles.

La pratique quotidienne de consultant nous conduit à constater qu'il y a bien relations entre l'ergonome consultant (le prestataire) et l'usager (le client). L'une des caractéristiques de la relation de service est le déséquilibre entre le prestataire et l'usager, qui les installe dans une situation où ils vont être en mesure de produire à deux ce que ni l'un, ni l'autre ne pourrait réaliser seul.

1. Ces développements théoriques sont par ailleurs développés dans une thèse en cours de rédaction (Querelle).

2. Nous retenons la notion de système sociotechnique plutôt que celle d'entreprise. Cette notion se détache ainsi d'une définition juridique de l'entreprise qui pose un cadre trop restrictif. 
Si l'usager peut être défini comme l'acteur du système sociotechnique qui est à un instant $t$ en relation avec l'ergonome consultant alors une limite des précédentes définitions apparaît. En effet, l'ergonome consultant est confronté à un ensemble d'acteurs ou d'usagers avec qui il doit créer et entretenir autant de relations de service. La définition initiale qui proposait jusqu'ici une relation de service adressée à un unique client ne tient plus. Ainsi, les usagers sont extraits du système sociotechnique pour deux raisons. D'une part, leur réseau de relations se distingue alors de celui des autres acteurs à ce moment de la relation de service par l'interaction avec le prestataire; et d'autre part, la nature de ces relations avec le système sociotechnique se démarque de celle qu'ils ont au quotidien.

Nous proposons d'envisager l'intervention ergonomique comme un système au sens où Crozier et Friedberg l'entendent. C'est-à-dire " que le système est un ensemble, dont les parties sont interdépendantes, qui possède donc un minimum de structuration, ce qui le distingue du simple agrégat, et qui dispose, en même temps, de mécanismes qui maintiennent cette structuration et qu'on appellera mécanismes de régulation ». Les relations de services s'intègrent donc dans des «systèmes d'action concret » (Crozier, \& Friedberg, 1977, p. 244). Cette réflexion invite alors à envisager la relation de service plutôt comme un système de relations de services où les acteurs sont interdépendants les uns des autres et doivent se « rendre service » pour arriver à produire ensemble la réponse au problème rencontré.

\section{2.-Un système de relations de services comme modèle d'intervention ergonomique}

Envisager la relation de service comme un système de relations de services souligne l'interdépendance des éléments qui font système. Toute modification sur un des éléments aura des conséquences sur l'ensemble du système et réciproquement toute non modification possède ce même potentiel. Cette dimension apporte un élément de lecture sur la complexité du métier d'ergonome consultant et plus généralement du métier de conseil qui entre en relation de service avec non pas une organisation ou une entreprise, mais bien avec des usagers qui eux-mêmes agissent au sein d'un système sociotechnique qui nécessite des transformations (l'objet du service).

Le schéma initial pourrait alors évoluer ainsi :

Dans le schéma proposé (cf. Figure 3), une partie du travail de l'ergonome consultant relève de la gestion d'au moins six types de relations inhérentes à sa prestation à savoir :

- les relations des « usagers » avec le système sociotechnique à transformer comme celles relatives aux fonctions de management stratégique et opérationnel ;

- les relations des usagers entre eux dans leurs fonctions projet par exemple (chef de projet, concepteurs, responsable de l'industrialisation, ...);

- les relations des « usagers » avec l'ergonome consultant en tant que prestataire (donneur d'ordre, fournisseur d'éléments techniques, ...) ;

- les relations de l'ergonome consultant avec le système sociotechnique à transformer relevant du contenu de sa prestation (analyse de l'existant, élaboration de cahier des charges, simulations, ...);

- les relations de l'ergonome consultant avec les systèmes sociotechniques environnants qui spécifient son type de positionnement comme prestataire d'une entreprise de services, comme IPRP, comme expert CHSCT, etc. ;

- les relations du système sociotechnique à transformer avec les systèmes sociotechniques environnants définissant le contexte général de l'intervention de l'ergonome consultant (par exemple, une intervention ergonomique dans le cadre d'un plan social diffère complètement d'une intervention au sein d'un projet 
d'investissement).

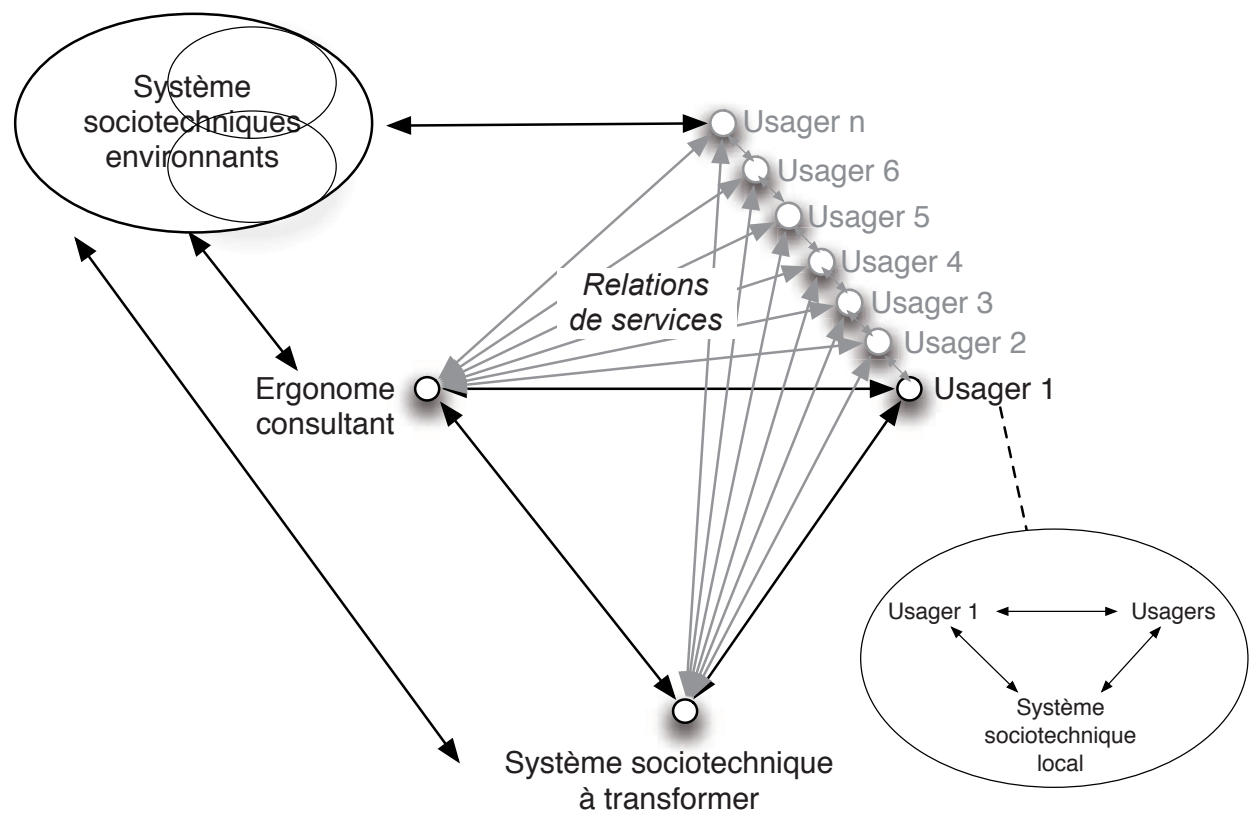

Figure 3 : Système de relations de services

Dans cette perspective, la co-construction (prestataire usagers) du positionnement de l'ergonome consultant au sein d'une conduite de projet industriel et/ou architectural se structure entre autres par ces six types de relations de services. Par exemple, les fonctions de management stratégique et opérationnel associées aux quatre familles de critères de gestion des projets (Thibault, 2000) structurent ce modèle de relations de services que l'ergonome consultant va mettre en œuvre au cours de son intervention ergonomique.

La perspective de construction sociale (Thibault, 2002 ; Daniellou, 2005) va immanquablement multiplier le nombre d'usagers de l'intervention ergonomique et par conséquent multiplier d'autant les types de relations de services. De plus, elles ne préexistent pas à l'intervention. Elles se constituent au fur et à mesure de l'avancée du service.

\section{3.-Dimension temporelle et outils intermédiaires}

Comme toute démarche constructive, l'intervention ergonomique se déroule dans un espace temporel jalonné d'étapes. En effet, l'intervention de l'ergonome consultant diffère en fonction de la temporalité du projet. De plus, du début à la fin de l'intervention les usagers vont évoluer, le plus souvent de nouveaux apparaissent modifiant les situations de gestion.

Cette dimension temporelle renvoie d'une part à un aspect dynamique et évolutif des relations de services. En effet, toute tentative de représentation d'une intervention par ce dispositif ne peut être valable qu'à un instant $\mathrm{t}$.

D'autre part, cette dimension temporelle renvoie aussi à la notion de système sociotechnique à transformer. En effet, si «l'objet final » du service est la transformation ou la conception d'un système sociotechnique, il n'est jamais directement l'objet de travail pendant l'intervention. En effet, comme le décrivent Jeantet, Tiger, Vinck et Tichkiewitch (1996), la conception repose sur des objets intermédiaires qui vont permettre d'amener l'ensemble ou une partie des usagers à travailler sur l'ensemble ou une partie des éléments à concevoir. 


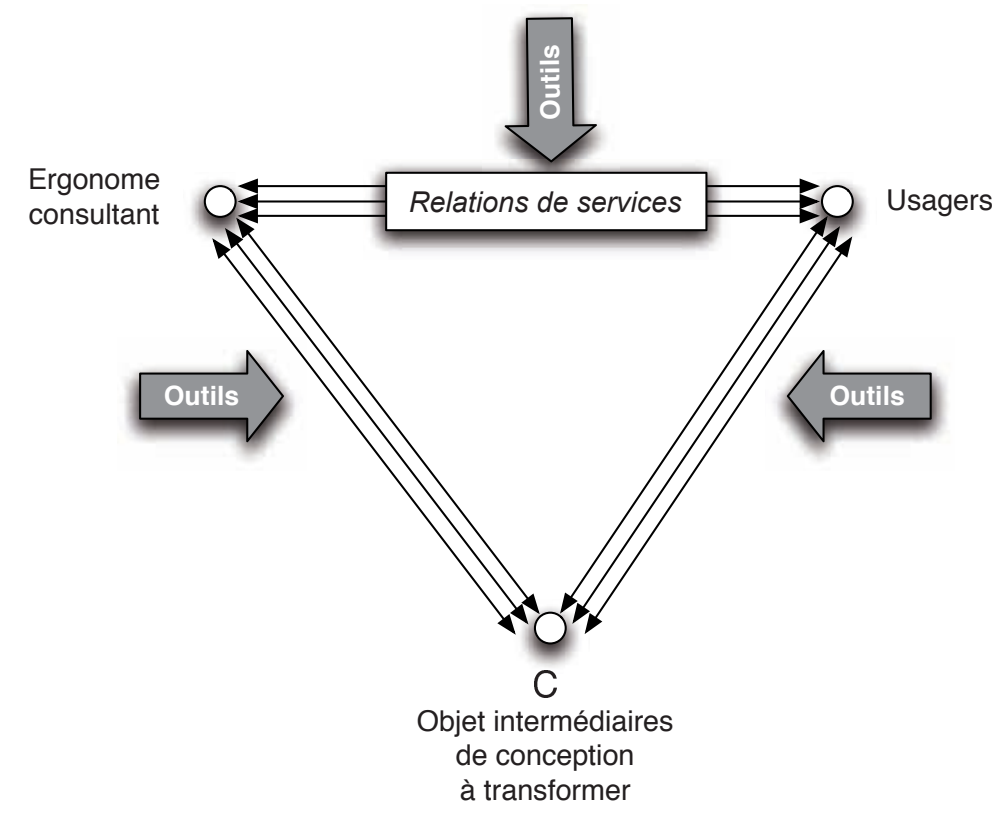

Figure 4 : Proposition de modélisation de l'intervention ergonomique

Nous proposons une ébauche de représentation de l'intervention ergonomique vue comme un système (cf. Figure 4). "L'intervention système » se déploie à la fois à l'intérieur et à l'extérieur du système sociotechnique. Pour partie, elle se réalise dans le temps et le lieu de l'entreprise concernée, mais elle est marquée par toute l'expérience du consultant voire de l'équipe de consultants. Pour une autre partie, elle est préparée à l'extérieur de l'entreprise. Il est possible de faire un parallèle entre ce qui lie ces deux « temps » et le lien « front-office», « back-office» discuté par Petit (2005).

L'intervention ergonomique n'a d'effet que sur une partie du projet. Les décideurs du système sociotechnique (PDG, directeurs industriels, chefs de projet, ...) sont les acteurs qui conservent la décision finale dans l'intervention. Des acteurs internes (opérateurs, cadres de proximité,...) vont être mobilisés comme ressource dans l'intervention ce qui génère des effets sur l'ensemble du système sociotechnique. Le projet reste un élément de l'entreprise soumis à l'influence directe de l'ensemble des acteurs internes.

L'ergonome consultant est un acteur externe parmi d'autres avec lesquels il doit souvent coopérer. Il conçoit ou transforme des objets intermédiaires adaptés à la situation et aux objectifs de l'intervention. Si ces objets intermédiaires de conception sont classiquement des schémas, des plans, des cahiers des charges, ..., ceux-ci peuvent être aussi entièrement conçus par l'ergonome consultant lors de son intervention.

\section{3.- Exemples d'outils utilisés par l'ergonome consultant}

L'ergonome consultant met en œuvre plusieurs types d'outils structurant le système de relations de services, nous avons retenu ici l'exemple d'un outil de gestion de projet.

\subsection{Deux catégories d'outils de l'ergonome consultant dans le système de relations de services}

Dans notre pratique d'ergonome consultant, nous utilisons des outils qui vont structurer notre prestation (cf Figure 5), (Thibault, 2002; Querelle, \& Escouteloup, 2005). À partir de la modélisation précédemment explicitée, ces outils peuvent être classés en deux catégories : 
- les outils support aux relations de services ;

- les outils support à la structuration des objets intermédiaires de conception.

Bien évidemment, ces outils sont évolutifs et s'inscrivent temporellement dans le projet en fonction des usagers et des objets de conception intermédiaires. Ces outils permettent de fournir des repères aux usagers à la fois de type :

- prescriptif (par exemple, intégration dans les cahiers des charges de référence à des normes en ergonomie) ;

- descriptif (par exemple, simulations de situations de travail et mise à jour de leurs déterminants) ;

- procédural (par exemple, planning de l'intervention ergonomique coordonnée au planning général du projet).

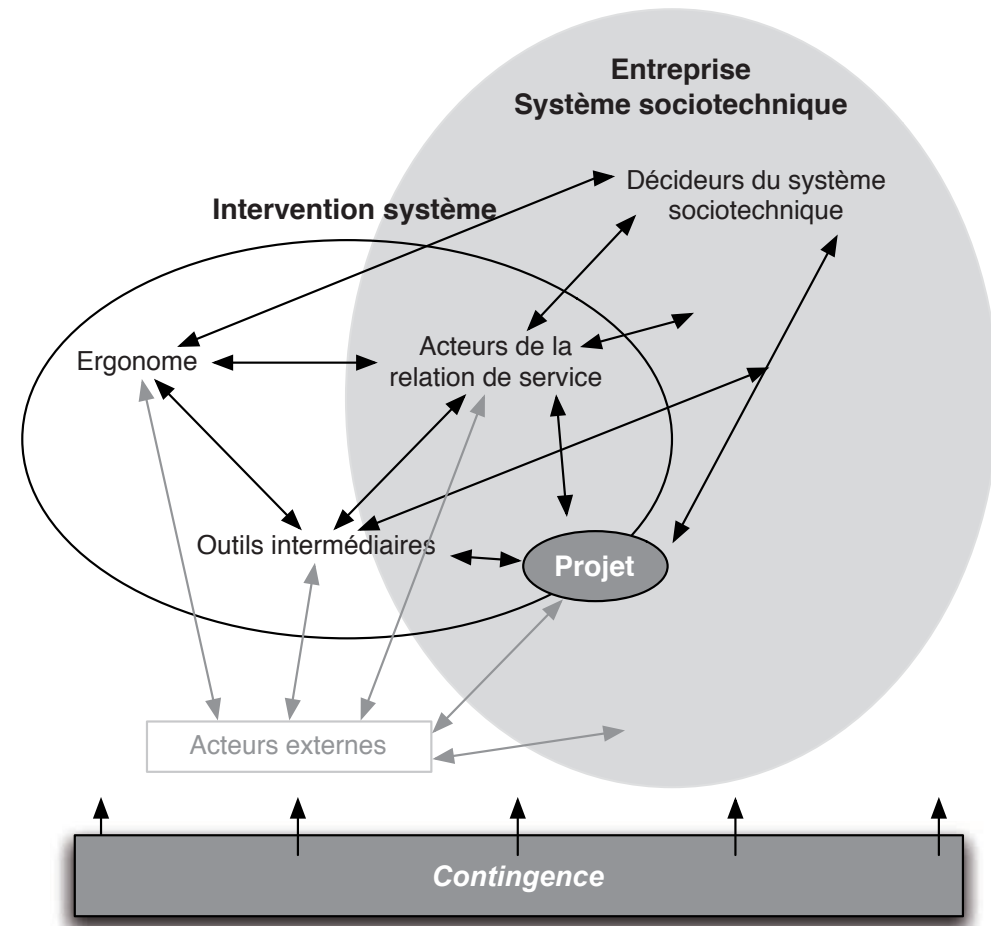

Figure 5 : Les outils intermédiaires de l'ergonome consultant dans le système de relations de services

Ainsi, ils assurent aussi une forme de traçabilité de l'intervention ergonomique et par là même le support d'une évaluation de la performance de l'intervention.

Nous inscrivons donc l'utilisation d'outils par l'ergonome consultant dans un modèle d'évaluation de la performance de l'intervention ergonomique (Thibault, 2002) dans la mesure où ceux-ci :

- sont collectivement partagés constituant un référentiel opératif commun ;

- s'inscrivent dans une dynamique de construction sociale dans et par l'intervention ergonomique ;

- sont construits, validés et utilisés comme un des indicateurs de performance ;

- sont médiateurs dans les relations entre le management stratégique et opérationnel du projet. 
En effet, l'ergonome consultant gère l'évolution de ses outils au fil du déroulement de son intervention ergonomique. Il inscrit sa démarche au cœur d'une intégration transversale des informations et des modes de communication, structurant les relations de services et les objets intermédiaires de conception. Nous rejoignons là une fonction fondamentale de la gestion de projet en ingénierie simultanée à savoir « l'intégration par les données » (Durand, 2004) afin que les mêmes informations soient partagées et échangées entre les différentes fonctions de l'entreprise.

\section{2.-Illustration par un outil de gestion}

Les outils de simulations utilisés par l'ergonome (plans, maquettes, etc.) ont fait l'objet de nombreuses recherches et publications qui montrent l'importance de cette catégorie d'outil en tant qu'objet intermédiaire de la conception. Nous avons donc choisi d'illustrer notre propos en présentant plutôt un outil de gestion de projet : «le document de suivi de projet industriel ». Cet outil utilisé en intervention nous permet de structurer nos relations de services avec les différents acteurs du projet.

\begin{tabular}{|l|c|l|c|}
\hline \multicolumn{4}{|c|}{ Tâche : partage des fillets } \\
\hline $\begin{array}{c}\text { Situation de } \\
\text { travail }\end{array}$ & \multicolumn{1}{|c|}{ Remarques } & $\begin{array}{c}\text { Repères pour la } \\
\text { conception }\end{array}$ & État* $^{*}$ \\
\hline $\begin{array}{l}\text { Aiguisage } \\
\text { des couteaux }\end{array}$ & $\begin{array}{l}\text { L'usure de la lame varie } \\
\text { en fonction du geste et } \\
\text { de la dureté de la chair } \\
\text { du filet }\end{array}$ & $\begin{array}{l}\text { Création d'une zone } \\
\text { dédiée à procimité de } \\
\text { la salle de parage }\end{array}$ & $\mathrm{R}$ \\
\hline
\end{tabular}

*: $R$ : réalisé, $E$ : étude en cours ; $A$ : abandon

Figure 6 : Document de suivi

Le document ${ }^{3}$ de suivi de projet industriel (cf. Figure 6) est rédigé et maintenu à jour par l'ergonome tout au long de l'intervention ergonomique. La structure du document reprend les différentes situations de travail classées par secteur, ou par atelier. Il est basé sur l'analyse du travail effectué par l'ergonome consultant intégrant à la fois les données issues de l'analyse de situations de travail existantes mais aussi de l'analyse des déterminants des futures situations de travail. Ce document est utilisé aussi bien dans un projet relatif à des transformations sur un site existant, que dans un projet de conception de nouvelles installations.

L'intérêt majeur de ce type d'outil est de structurer et partager l'information :

- Il permet à chacun des acteurs présents ou non pendant les réunions de garder une visibilité sur l'avancée des décisions de conception et d'identifier les points restant à travailler. Les acteurs ont ainsi la possibilité d'interpeller l'intervenant et d'anticiper la préparation des travaux à venir.

- Lors de ces phases de préparation, les acteurs ont connaissance de la manière dont l'information va être présentée et utilisée. Le tableau est donc facilitateur lors des phases de préparation et de simulation avant instruction. Ensuite, lors des phases d'instruction, les décisions sont éclairées par l'ensemble des éléments relevés par les acteurs de l'entreprise et le prestataire.

- Cet outil permet aussi à l'intervenant qui participe à plusieurs projets en même temps de facilement reprendre le cours de la réflexion dans la mesure où il lui suffit en fonction du point abordé d'ouvrir le tableau au thème concerné et de contrôler l'ensemble des décisions prises et des points à travailler.

- Le tableau de bord diffusé à chaque réunion permet aussi à l'information de circuler

3. Pour plus de précisions sur cet outil, voir la présentation de Thibault (2002) au congrès de la SELF. 
dans l'entreprise en dehors des temps de présence de l'ergonome. Ce qui permet aux acteurs absents ou aux instances représentatives de toujours conserver une lisibilité sur l'avancée des décisions, leurs implications et leurs limites.

Ce tableau ouvre des perspectives d'évaluation. Il permet en effet de comptabiliser le nombre d'éléments étudiés dans le projet et de le comparer au nombre d'éléments mis en œuvre par les décisionnaires. C'est une des traces de l'activité de travail de l'ergonome qui permet de quantifier et de qualifier les effets de sa prestation en regard du coût de son intervention.

Ce type d'outil structure les relations de services que l'ergonome consultant entretient avec les différents secteurs de l'entreprise (exploitant, ressources humaines, ingénierie, méthodes, sécurité, etc.) et avec les intervenants externes de l'entreprise (fournisseurs divers, architecte, bureau de contrôle, etc.) afin que chacun à son niveau puisse intégrer les informations relatives à l'intervention en ergonomie. Avec le support des outils informatiques, ce document est aussi facilement transmis, modifiable, transformable, autrement dit : appropriable. Ainsi, il devient régulièrement dans nos interventions le document de l'entreprise sur lequel le chef de projet coordonne les différents niveaux d'affectation des tâches, de délais, de prise de décision.

\section{4.- Conclusion}

Dans l'intervention, l'ergonome consultant est confronté à une quantité importante d'acteurs « usagers » mobilisés, ce qui d'une part engendre des difficultés vis-à-vis du nombre de variables à gérer, mais d'autre part contribue à la performance de l'intervention ergonomique. Ainsi, la coproduction de relations de services et d'objets intermédiaires appelle obligatoirement un certain nombre d'acteurs nécessaires. L'ergonome consultant doit donc en permanence gérer les dimensions individuelles et collectives, alors qu'elles sont toutes deux à la fois ressources et difficultés. De plus, elles ne se « travaillent» pas de la même manière. Il doit mettre en œuvre des outils qui, tout au long de leur cycle d'utilisation, vont médiatiser le processus de conception et organiser les rapports sociaux entre les acteurs.

Cette recherche en termes de pratique réflexive, montre aussi qu'en observant le métier d'ergonome consultant sous l'angle d'un système de relations de services, il apparaît que ce métier relève autant du métier de conseil que du métier d'ergonome. Bien entendu, ce constat de la double fonction ergonome-conseil en particulier dans des domaines non traités dans ce texte (comme le type de relation commerciale entretenue par l'ergonome consultant) mérite d'être approfondi. Ainsi cet angle de compréhension de l'intervention ergonomique laisse présager de nombreuses applications pédagogiques dans la formation au métier d'ergonome consultant.

\section{RÉFÉRENCEMENT}

Querelle, L., \& Thibault, J.-F. (2007). La pratique de l'intervention d'ergonomes consultants : une approche réflexive orientée par les outils. @ctivités, 4 (1), pp 149-159, http://www.activites.org/v4n1/v4n1.pdf.

\section{RÉFÉRENCES}

Cerf, M., \& Falzon, P. (Eds.) (2005). Situations de service : travailler dans l'interaction. Paris: PUF.

Crozier, M., \& Friedberg, E. (1977). L'acteur et le système. Paris: Seuil.

Daniellou, F. (2005). The French-speaking ergonomists' approach to work activity: cross-influences of field intervention and conceptual models. Theoretical Issues in Ergonomics Science, 6 (5), 405-427

Durand, J.P. (2004). La châ̂ne invisible. Paris: Seuil. 
Falzon, P., \& Lapeyrière, S. (1998). L'usager et l'opérateur : ergonomie et relation de service. Le travail humain, 61 (1), 69-88.

Gadrey, J. (1994). Les relations de service dans le secteur marchand. In J. de Bandt, \& J. Gadrey (Eds.), Relations de service, marchés de services (pp. 23-41). Paris: CNRS Éditions.

Goffman (1968). Asiles. Paris: Éditions de Minuit.

Hubault, F. (Ed.) (2001). La relation de service, opportunités et questions nouvelles pour l'ergonomie. Toulouse: Octarès Éditions.

Jackson, M. (1998). Entre situations de gestion et situation de délibération : l'action de l'ergonome dans les projets industriels. Thèse de doctorat d'Ergonomie. Paris: Laboratoire d'Ergonomie du CNAM.

Jeantet, A., Tiger, H., Vinck, D., \& Tichkiewitch, S. (1996). La coordination par les objets dans les équipes intégrées de conception de produits. In G. de Terssac, \& E. Friedberg (Eds.), Coopération et conception (pp. 87-100). Toulouse: Octarès Éditions.

Mayen, P. (2005). Travail de relation de service, compétences et formation. In M. Cerf, \& P. Falzon (Eds.), Situations de service : travailler dans l'interaction (pp. 61-83). Paris: PUF.

Petit, J. (2005). Organiser la continuité du service: Intervention sur l'organisation d'une Mutuelle de santé. Thèse de doctorat d'Ergonomie. Bordeaux: Université Victor Segalen Bordeaux 2.

Querelle, L., \& Escouteloup, J. (2005). The tribulations of an ambulatory sector. In R. Tartaglia, \& S. Bagnara (Eds.), Healthcare systems ergonomics and patient safety: Human Factor, a bridge between care and cure (p.314-317). Londres: Taylor \& Francis.

Thibault, J.-F. (2000). Practice of ergonomics management in industrial design. In "Ergonomics for the new millennium”, IEA 2000, XIVth Triennal congress and human factors and ergonomics society, San Diego, USA, pp.297-299.

Thibault, J.-F. (2002). Quand le processus de prescription reflète la performance de l'intervention ergonomique. In J.-M. Evesque, A.-M. Gautier, Ch. Revest, Y. Schwartz, \& J.-L. Vayssière (Eds.), Les évolutions de la prescription (pp. 370-375). Aix-en-Provence: GREACT et SELF.

Villette, M. (2003). Sociologie du conseil en management. Paris: Éditions La Découverte (Collection Repères).

\section{RÉSUMÉ}

Cet article propose d'analyser le travail de l'ergonome consultant en intervention en partant d'un modèle théorique de la relation de service. En effet, les auteurs de ce modèle montrent que l'activité de conseil repose sur une relation de service à part entière. Dans cette perspective, il s'avère que la relation de service appliquée à l'intervention ergonomique ouvre la discussion sur les objets utilisés dans l'intervention. Cependant il est nécessaire d'adapter les contours théoriques de la relation de service pour étudier l'intervention ergonomique. C'est donc à partir d'une démarche réflexive sur la pratique de l'intervention par des ergonomes consultants que nous avons éprouver la dimension heuristique d'un tel modèle et esquisser de nouvelles perspectives.

\section{Mots Clé}

théorie de l'activité, relation de service, conduite de projet, modèle d'intervention ergonomique

\section{RESUMEN}

\section{La práctica de la intervención de los ergónomos consultores: une}


enfoque reflexivo orientado por las herramientas. Este artículo se propone analizar el trabajo del ergónomo consultor que realiza intervenciones, partiendo de un modelo teórico de la relación de servicio. En efecto, los autores de este modelo muestran que la actividad de consultoría reposa enteramente sobre una relación de servicio. En este sentido, se constata que la relación de servicio aplicada a la intervención ergonómica abre la discusión acerca de los objetos utilizados en la intervención. Sin embargo, para estudiar la intervención ergonómica, se hace necesario adaptar los contornos teóricos de la relación de servicio. Por lo tanto, será a partir de un acción reflexiva acerca de la práctica de intervención de los ergónomos, que pondremos a prueba la dimensión heurística de tal modelo, así como bosquejar nuevas perspectivas.

Palabras-clave

Teoría de la actividad, relación de servicio, conducción de proyectos, modelo de intervención ergonómica. 